\title{
Third and fourth heart sounds had low sensitivity but moderate to high specificity for predicting left ventricular dysfunction.
}

Marcus GM, Gerber L, McKeown BH, et al. Association between phonocardiographic third and fourth heart sounds and objective measures of left ventricular function. JAMA 2005;293:2238-44.

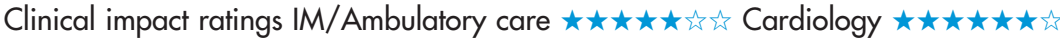

In patients referred for non-emergent left sided heart catheterisation, how accurate are the third $\left(S_{3}\right)$ and fourth $\left(S_{4}\right)$ heart sounds detected by computerised phonocardiography for predicting left ventricular dysfunction?

\section{METHODS}

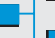

Design: blinded comparison of $\mathrm{S}_{3}$ and $\mathrm{S}_{4}$ with $\mathrm{B}$ type natriuretic peptide (BNP) concentration, left ventricular end diastolic

pressure (LVEDP), and left ventricular ejection fraction (LVEF) as reference standards.

(र्थ

Setting: a university teaching hospital in the US.

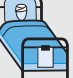

Patients: 90 patients (mean age 62 y, 61\% men) who were referred for elective left sided heart catheterisation. Exclusion criteria included age $<18$ years and systolic pressure $<90 \mathrm{~mm} \mathrm{Hg}$.

Description of test: $S_{3}$ and $S_{4}$ were obtained from

1. [1] audioelectrocardiographic data generated by the Audicor System (Audicor, Inovise Medical Inc, Portland, OR, USA), with the audioelectrocardiographic leads attached to $V_{3}$ and $V_{4}$ positions and connected to a Marquette MAC 5000 (Genera Electric Healthcare Technologies, Waukesha, WI, USA).

Diagnostic standards: BNP concentrations measured using a membrane immunoflourescence assay (Biosite Inc, San Diego, CA, USA) with BNP concentrations $>100 \mathrm{pg} / \mathrm{ml}$ specified as abnormal; LVEDP recorded using a $6 \mathrm{~F}$ pigtail catheter and a fluid filled pressure transducer, with an LVEDP $>15 \mathrm{~mm} \mathrm{Hg}$ specified as abnormal; and LVEF measure obtained using transthoracic echocardiography (Acuson Sequoia, Mountain View, CA, USA or SONOS 5500, Philips Medical Systems, Andover, MA, USA). An LVEF $<50 \%$ was defined as abnormal.

Outcomes: sensitivity and specificity, and positive and negative likelihood ratios calculated from data in the article.

\section{MAIN RESULTS}

$46 \%$ of patients had abnormal LVEDP, $28 \%$ abnormal LVEF, and $58 \%$ abnormal BNP. The table shows sensitivity, specificity, and positive and negative likelihood ratios of $S_{3}$ and $S_{4}$.

\section{CONCLUSION}

In patients referred for non-emergent left sided heart catheterisation, third and fourth heart sounds detected by phonocardiography had low sensitivity but moderate to high specificity for predicting left ventricular dysfunction.
For correspondence: Dr Andrew D Michaels, University of California San Francisco Medical Center, San Francisco, CA USA. andrewm@medicine. ucsf.edu

Source of funding: National Institutes of Health Mentored Patient-Oriented Research Career Development Award.

\section{Commentary}

he study by Marcus et al represents a gold standard approach for determining the accuracy of physical exam findings. By using an objective, unbiased, and reproducible phonocardiographic instrument, this study of the long held physical exam findings of an $\mathrm{S}_{3}$ and $\mathrm{S}_{4}$ for detecting left ventricular dysfunction showed only modest diagnostic accuracy with low sensitivities and area under the receiver operating characteristic curve. The authors performed a study that could be easily validated elsewhere and in other populuations, setting the standard for studies of physical exam findings.

While the importance of these physical exam findings has been taught for decades, no study has determined their sensitivity and specificity using reproducible methods. Most studies rely on human detection, which is often variable and usually in the context of highly experienced physicians. Several studies lack blinding to patient's clinical condition, or the prevalence of left ventricular dysfunction is expected to be high.

Although the diagnostic accuracy of an $\mathrm{S}_{3}$ is modest, previous studies have shown that detection of an $\mathrm{S}_{3}$ is associated with a worse prognosis. It is important to note that, as in other studies, the present study showed that detection of an $\mathrm{S}_{3}$ had a high specificity for predicting left ventricular dysfunction. Thus, the cardiovascular exam should still continue to include the auscultation for gallops, but as a characterisation for the degree of cardiovascular disease present. The absence of a gallop should not be used to indicate the absence of left ventricular dysfunction and certainly cannot replace other diagnostic testing when screening for left ventricular dysfunction is indicated.

With any study, important limitations can be discerned. The exclusion criteria were reasonable and aimed to provide a more homogeneous population, but patients with mitral regurgitation or stenosis, serum creatinine concentration $\geqslant 4.0 \mathrm{mg} / \mathrm{dl}$, and severe pulmonary hypertension should be excluded when applying the results. Because the study enrolled patients referred to a cardiac catheterisation lab, the prevalence of left ventricular dysfunction is likely higher than in other populations. Thus, results from this study may be different in other populations.

Adrian F Hernandez, MD Duke University Medical Center Durham, North Carolina, USA

Diagnostic properties of the third $\left(S_{3}\right)$ and fourth $\left(S_{4}\right)$ heart sounds detected by phonocardiography for predicting left ventricular dysfunction*

\begin{tabular}{llllll}
\hline $\begin{array}{l}\text { Heart } \\
\text { sound }\end{array}$ & $\begin{array}{l}\text { Gold } \\
\text { standard }\end{array}$ & $\begin{array}{l}\text { Sensitivity } \\
\text { (95\% Cl) }\end{array}$ & Specificity (CI) & +LR & - LR \\
\hline S3 & LVEDP & $41 \%(26$ to 58$)$ & $92 \%(80$ to 98$)$ & 5.13 & 0.64 \\
& LVEF & $52 \%(31$ to 73$)$ & $87 \%(76$ to 94$)$ & 4.00 & 0.55 \\
& BNP & $32 \%(20$ to 46$)$ & $92 \%(78$ to 98$)$ & 4.00 & 0.74 \\
S4 & LVEDP & $46 \%(31$ to 63$)$ & $80 \%(66$ to 90$)$ & 2.30 & 0.68 \\
& LVEF & $43 \%(23$ to 66$)$ & $72 \%(59$ to 82$)$ & 1.54 & 0.79 \\
& BNP & $40 \%(26$ to 54$)$ & $78 \%(61$ to 90$)$ & 1.82 & 0.77 \\
S3 and/ & LVEDP & $68 \%(52$ to 82$)$ & $73 \%(59$ to 85$)$ & 2.52 & 0.44 \\
or S4 & LVEF & $74 \%(52$ to 90$)$ & $64 \%(52$ to 76$)$ & 2.06 & 0.41 \\
& BNP & $57 \%(42$ to 70$)$ & $72 \%(55$ to 86$)$ & 2.04 & 0.60 \\
\hline
\end{tabular}

*LVEDP = left ventricular end diastolic pressure; $L$ VEF = left ventricular ejection fraction; BNP = B type natriuretic peptide. Diagnostic terms defined in glossary. LRs calculated from data in article. 